\title{
Globe
}

Revue internationale d'études québécoises

\section{Howard Adelman et Pierre Anctil (dir.), Religion, Culture, and the State: Reflections on the Bouchard-Taylor Report, Toronto, University of Toronto Press, 2011}

\section{Rachad Antonius}

Volume 15, numéro 1-2, 2012

URI : https://id.erudit.org/iderudit/1014649ar

DOI : https://doi.org/10.7202/1014649ar

Aller au sommaire du numéro

Éditeur(s)

Globe, Revue internationale d'études québécoises

ISSN

1481-5869 (imprimé)

1923-8231 (numérique)

Découvrir la revue

Citer ce compte rendu

Antonius, R. (2012). Compte rendu de [Howard Adelman et Pierre Anctil (dir.), Religion, Culture, and the State: Reflections on the Bouchard-Taylor Report,

Toronto, University of Toronto Press, 2011]. Globe, 15(1-2), 370-373.

https://doi.org/10.7202/1014649ar d'utilisation que vous pouvez consulter en ligne.

https://apropos.erudit.org/fr/usagers/politique-dutilisation/ 
Dans sa conclusion, Yvan Lamonde passe de la parole de l'historien à celle du citoyen. Son opposition à la laïcité ouverte du rapport Bouchard-Taylor et au port des symboles religieux dans la fonction publique est exposée dans les derniers chapitres. Il manque toutefois, à mon avis, un pont plus explicite entre les débats actuels et ceux des années 1960 autour de l'éducation. Car bien qu'ils ne s'entendent pas sur la définition et les effets pratiques de la laïcité et de la neutralité de l'État, les protagonistes actuels revendiquent le même mot, laïcité, et le même héritage, la Révolution tranquille. Lamonde aurait pu détailler davantage les raisons qui le conduisent à percevoir dans une neutralité plus affirmée de l'État et de l'espace public, sans symboles ostentatoires, la continuité de la tradition de la laïcité, contrairement aux dires des partisans d'une laïcité ouverte. Cette critique ne remet aucunement en question la qualité de l'ouvrage et la grande perspicacité de son auteur. À preuve, la référence aux propos de Maurice Blain, datant des années 1960, comme quoi la marche vers la laïcité comporte avec elle une "obligation de reconstruire quelque forme de consensus ». Or c'est bien aujourd'hui la question de ce consensus (culture civique commune, consensus par recoupement, tradition) à laquelle se butent les différents protagonistes de la laïcité. Yvan Lamonde rappelle ici, dans un essai riche et fécond, que l'une et l'autre de ces questions sont inséparables.

Bernard Gagnon Université du Québec à Rimouski

\section{Howard Adelman et Pierre Anctil (dir.) Religion, Culture, and the State: Reflections on the Bouchard-Taylor Report, Toronto, University of Toronto Press, 2011.}

Il s'agit d'un ouvrage collectif publié sous la direction des deux auteurs principaux. L'ouvrage est dédié à Ralph Halbert, fondateur du Halbert Center for Canadian Studies de l'Université hébraïque de Jérusalem, en Cisjordanie.

Chacun des chapitres amène des éléments empiriques importants concernant les débats soulevés par la commission Bouchard-Taylor et le rapport entre la religion, la culture et l'État. Ces questions sont problématisées à travers une série de tensions, qui structurent le matériau empirique 
mobilisé : les tensions qui se sont manifestées dans l'histoire entre les anglophones et les francophones; la tension entre la reconnaissance des spécificités identitaires des groupes et la recherche de valeurs communes; la tension entre la réalité de l'insertion des communautés juives dans l'histoire québécoise et le statut d'altérité qu'elles ont eu et ont toujours; l'opposition entre des conceptions dites "ouvertes" de la laïcité et celles dites "rigides", telles qu'elles se manifestent dans les décisions de la Cour suprême du Canada et dans les positions de la Commission; la tension entre les attitudes exprimées face à l'altérité (plutôt hostiles) et les comportements dans le quotidien, plutôt tolérants et pragmatiques; et la tension entre l'individualisme et le communautarisme.

Malgré la riche matière empirique qu'on y trouve, l'entreprise reste prisonnière d'une orientation générale que l'on ressent, mais qui n'est pas explicitée, d'un paradigme implicite, pourrait-on dire, que nous allons tenter de cerner.

Dans son chapitre introductif, Pierre Anctil montre que la politique québécoise de l'interculturalisme a été construite sur les bases de la politique fédérale du multiculturalisme, mais en y ajoutant des éléments qui garantiraient l'intégration des immigrants à la majorité francophone du Québec. Il rappelle que la Charte canadienne des droits et libertés, promulguée en 1982, ne fait aucune référence à une quelconque laïcité de l'État canadien, ni à aucune obligation qui serait faite aux fonctionnaires fédéraux de respecter une neutralité absolue par rapport à la religion.

Il souligne que, «alors que cette loi canadienne fondamentale a été mise en œuvre en premier lieu pour protéger les individus contre l'arbitraire de l'État, nous commençons à voir à présent qu'elle peut aussi indirectement appuyer des revendications d'une nature communautariste ou en lien avec les identités minoritaires» (p. 27).

Anctil estime que la Commission a contribué à clarifier le sens des accommodements et a rappelé à la majorité sa responsabilité dans l'établissement d'un climat propice au dialogue, mais qu'elle n'a pas été capable de traiter adéquatement de la question de la laïcité. Il termine son chapitre en déplorant que la commission Bouchard-Taylor n'ait pas été capable de contribuer au débat de façon novatrice et qu'elle n'ait pas contribué à la résolution de difficultés spécifiques. Il estime que les commissaires ont quelquefois manqué de sens politique en ne se dissociant pas clairement de remarques hostiles faites durant les audiences, mais que dans le concret du quotidien, les Québécois gèrent plutôt bien la question du pluralisme qui les entoure. 
Pour sa part, Howard Adelman met en parallèle la situation française et celle du Québec par une comparaison entre la commission Stasi et la commission Bouchard-Taylor. Il exprime des vues assez critiques sur la société québécoise, en ironisant, par exemple, sur le fait que le Québec parle d'accommodements raisonnables, mais que les outils mis en place par l'État pour protéger la langue sont complètement déraisonnables (p. 51). Il souligne que si la commission Stasi a bien été accueillie en France, la commission Bouchard-Taylor a été sévèrement critiquée par le mouvement souverainiste au Québec et qu'elle l'aurait été encore plus si elle avait révélé les aspects sombres de ce mouvement.

Ira Robinson se penche spécifiquement sur la façon dont la commission Bouchard-Taylor a abordé les relations entre la communauté juive et les Québécois et se félicite du fait que la commission ait remis les pendules à l'heure en corrigeant certaines perceptions fausses mais généralisées dans la société québécoise concernant la communauté juive. Il conclut que les Juifs restent les représentants de l'altérité pour la plupart des Canadiens français et reprend à son compte l'évaluation d'un chroniqueur de The Gazette qui déplore que le nationalisme ethnique soit redevenu respectable au Québec.

Bina Toledo Freiwald propose une analyse qualitative du contenu des mémoires présentés à la commission, pour conclure que le débat fondamental se fait dans le cadre d'un discours fondé sur la nation. Elle affirme que les valeurs universelles affichées par les citoyens québécois palissent devant l'attrait puissant des récits identitaires et des promesses d'appartenance qu'ils portent, et conclut que le débat est entre deux modèles de la communauté politique : celui de la nation fondée sur un peuple (peoplehood), et celui de la citoyenneté (p. 85).

José Woehrling montre que les conceptions de la laïcité défendues par la Commission rejoignent celles de la Cour suprême du Canada en ce qu'elles s'apparentent à une laïcité dite "ouverte": neutralité religieuse stricte des institutions de l'État et de ses politiques, mais permission pour les individus qui y travaillent de manifester leurs convictions ou leurs appartenances.

Dans sa conclusion, Adelman approfondit la réflexion sur les tensions qui se manifestent dans la philosophie politique entre l'individualisme et le communautarisme. Il propose une argumentation qui vise à dépasser l'opposition entre ces deux tendances, estimant qu'elles peuvent se rejoindre, si on y fait une place à la reconnaissance de la valeur et de la dignité de l'«autre». Il estime que la commission Bouchard-Taylor a démontré que «le problème au Québec est créé par des croyances et non pas par des pratiques» 
(p. 104) et souligne que les francophones au Canada sont fondamentalement inquiets quant à leur identité et leur futur en tant que communauté.

Le ton du livre oscille entre un effort sérieux d'objectivité et un ton ironique que l'on décèle dans certains chapitres, légèrement condescendant envers la société québécoise et envers le mouvement souverainiste. Il est significatif, par exemple, qu'on trouve très peu de références à la riche littérature qui a été publiée en français au Québec sur l'interculturalisme, le multiculturalisme et la commission Bouchard-Taylor elle-même.

Il y a aussi, dans l'ensemble du manuel, une tendance à psychologiser les rapports politiques. C'est comme si la question de la diversité n'était problématique qu’à cause de l'insécurité identitaire des Québécois, qui est irrationnelle. "Aucune quantité de discours rationnel ne peut affecter cette sensibilité » (p. 104). La question des rapports de pouvoir réels, au sein de la fédération canadienne, qui peuvent avoir pour effet de marginaliser la société québécoise est totalement absente de l'analyse.

En conclusion, on peut estimer que l'ouvrage traduit les perspectives multiculturalistes de ce que signifie le débat sur les accommodements raisonnables au Québec, mais qu'il n'inclut pas de perspectives théoriques s'inspirant des courants nationalistes, qu'ils soient de tendance civique ou ethnique. Les intellectuels se réclamant de ces courants ont tout de même produit des analyses fort raisonnées, qu'on ne peut pas automatiquement assimiler à un nationalisme ethnique étroit. En d'autres termes, on pourrait dire que, de façon générale, l'ouvrage consiste en une mise en forme raisonnée des perceptions dominantes dans le ROC (Rest of Canada) des débats qui se sont développés autour de la commission Bouchard-Taylor. Il va sans dire que si cette perspective est partiale, c'est celle de la majorité canadienne, et qu'elle est portée par une partie de la communauté académique au Québec. Il serait intéressant de permettre aux lecteurs anglophones du Rest of Canada d'avoir aussi accès aux autres perspectives, celles des chercheurs qui intègrent dans leur approche théorique les sensibilités et les préoccupations de la nation québécoise, qui reste minoritaire au Canada.

Rachad Antonius

Université du Québec à Montréal 